\title{
A critical assessment of reconsolidation interference as a tool for therapeutic forgetting
}

\author{
Natalie Schroyens, Tom Beckers", Laura Luyten ${ }^{\#}$ \\ Centre for the Psychology of Learning and Experimental Psychopathology, Department of \\ Psychology, KU Leuven; Leuven Brain Institute; "equal contribution
}

\begin{abstract}
Re-exposure to elements of prior experiences can create opportunities for inducing amnesia for those events. The dominant theoretical framework posits that such re-exposure can result in memory destabilization, making the memory representation temporarily sensitive to disruption while it awaits reconsolidation. If veritable, a mechanism that allows for memories to be permanently changed could have important implications for the treatment of several forms of psychopathology. However, the way reconsolidation theory is currently formulated and applied to account for empirical data hampers scientific and clinical progress. Theoretical and methodological advances are essential for a fruitful translation of reminder-dependent amnesia into clinical treatment.
\end{abstract}

\section{Corresponding authors}

Tom Beckers at KU Leuven, Tiensestraat 102 bus 3712, 3000 Leuven, Belgium; Laura Luyten at KU Leuven, Tiensestraat 102 bus 3712, 3000 Leuven, Belgium

\section{E-mail addresses}

natalie.schroyens@kuleuven.be

tom.beckers@kuleuven.be

laura.luyten@kuleuven.be

\section{Acknowledgements}

We acknowledge the financial support of the European Research Council (ERC Consolidator Grant to T. Beckers, grant number 648176) and the Research Foundation - Flanders, Belgium (FWO Doctoral Fellowship to N. Schroyens, grant number 1114018N). 


\section{Introduction}

Emotional memories play an important role in several forms of psychopathology, including anxiety disorders, post-traumatic stress disorder (PTSD) and addiction. Targeting such unwanted memories is thus highly interesting from a clinical perspective (1). It has repeatedly been demonstrated in the lab that forgetting can be induced by combining a reminder to a previously acquired memory with any of a number of electrophysiological, pharmacological, genetic, or behavioral manipulations (2-4) (referred to as 'reminder-dependent amnesia' henceforth). However, translational studies in patient populations have yielded mixed results, suggesting that it is challenging to effectively apply reminder-dependent amnesia in clinical practice, and highlighting the need to advance our understanding of when, how and why amnesia may occur (1). We maintain that such theoretical progress is impeded by the way the theory is currently being formulated and empirically tested. After briefly introducing the theoretical framework for reminder-dependent amnestic effects, we elaborate on some of the issues that need to be resolved before considering clinical translation of reminder-dependent amnesia. Although we mainly focus on theoretically-oriented barriers and possible solutions, we also describe how methodological approaches can play an important role in moving the field forward.

The currently dominant theoretical framework posits that reminder-dependent amnestic effects rely on the occurrence of memory destabilization and reconsolidation. More specifically, reconsolidation theory assumes that a destabilized or active memory trace is temporarily sensitive to modification until the memory is presumably reconsolidated $(3,4)$ (Fig. 1). Several manipulations are thought to interfere with memory reconsolidation if administered while the memory is in an active state, resulting in a disrupted (or enhanced) memory. Functionally, it is assumed that reconsolidation serves to allow for the updating of memories by providing the opportunity for incorporating novel information into an existing memory trace (5).

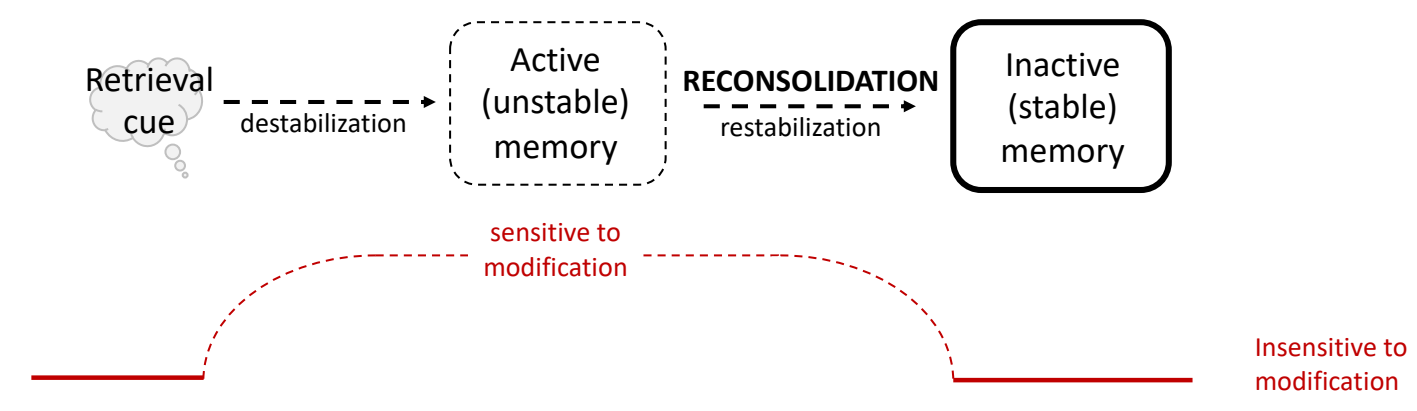

Figure 1. Schematic representation of reconsolidation theory. 
The introduction of reconsolidation as a theoretical framework has greatly promoted and inspired new research, and there is an abundance of empirical evidence that seems to be in accordance with the existence of a reconsolidation-like mechanism. Beyond initial findings with fear memories, the field has expanded enormously, including applications to different types of memory and using an extensive variation of manipulations in a wide range of species (6). As a result, the adoption of reconsolidation-based procedures has been touted as a highly promising approach for the clinical treatment of several forms of psychopathology.

Although reconsolidation theory has dominated the field, it has not been free from debate. It has been suggested that not all behavioral effects observed after combining a reminder with an amnestic manipulation can be attributed to reconsolidation interference (7-14). In addition, there has been a considerable amount of failures to conceptually or exactly replicate previously published studies that appeared to demonstrate memory reconsolidation (15-25) and reconsolidation theory presently cannot illuminate which factors explain those negative results.

Given the above-mentioned characteristics of the field (i.e., a 'hot' research area dominated by a single mechanistic theory, contradictory findings, several elusive occurrences of replication failures, and potential relevance for clinical applications), we believe that it is useful to critically evaluate the reconsolidation framework. The current review discusses how empirical behavioral studies relate to the process and theory of reconsolidation and describes how reconsolidation theory and its empirical validation could be improved (see Fig. 2 for an overview of the three main topics that we will address). In brief, reconsolidation theory's core assumptions entail that combining a reminder session with a manipulation can induce behavioral changes that rely on the occurrence of memory destabilization and reconsolidation (Fig 2, colored boxes). First, we will discuss the problem of reverse inference, i.e., attributing observed effects to the occurrence of unobservable processes such as destabilization or reconsolidation (Fig. 2, (1)). Second, we will argue that unconditional adherence to reconsolidation as the underlying mechanism of observed results affects the way reconsolidation theory has been used to explain and predict behavioral effects (Fig. 2, (2)). Third, we will elaborate on how the use of auxiliary assumptions, consisting of a range of vaguely-defined boundary conditions, has allowed researchers to explain most possible outcomes with recourse to reconsolidation theory and protect this theory from refutation (i.e., auxiliary assumptions form a 'protective belt' around the theory's core assumptions; Fig. 2, (3)) (26). 


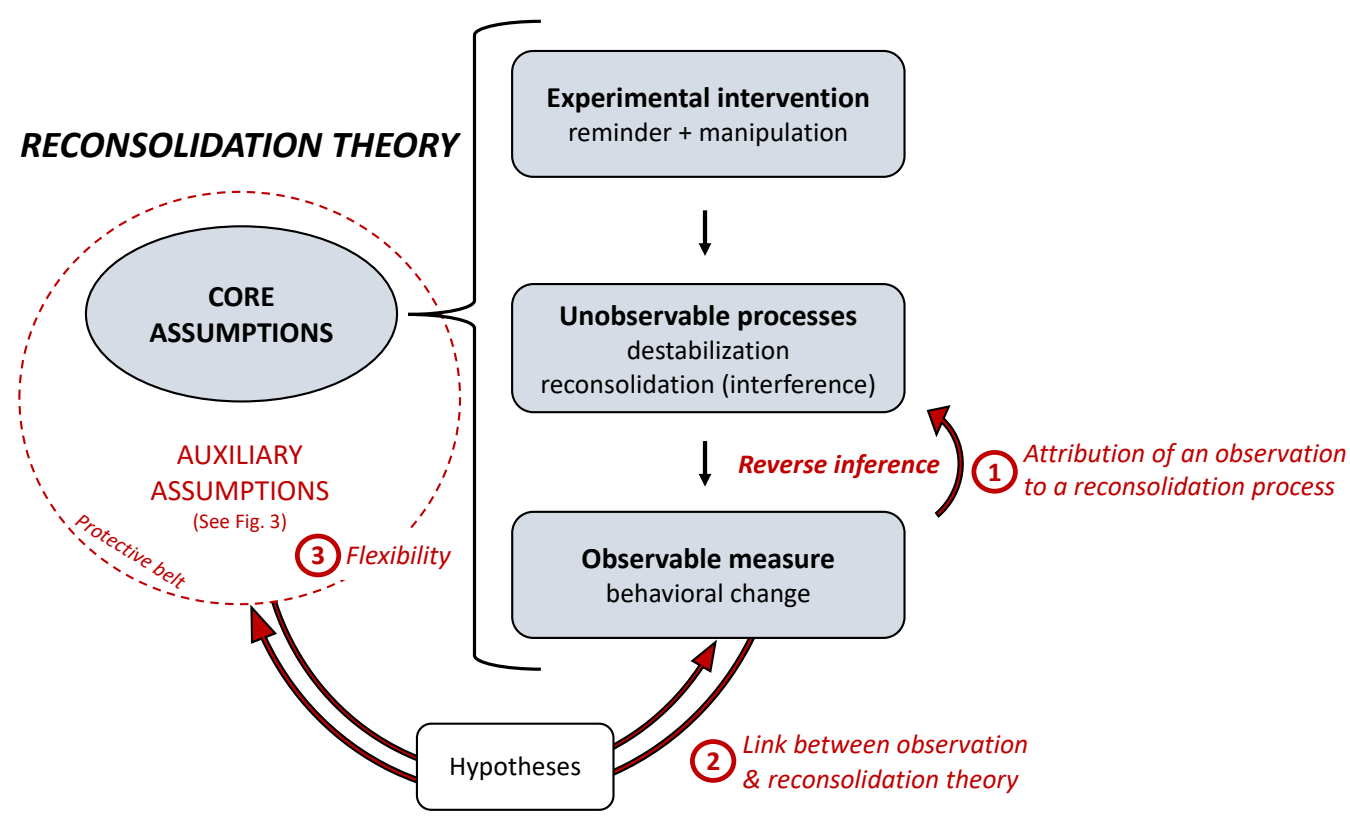

Figure 2. The current review evaluates how empirical observations have been related to (1) the unobservable process and (2) the theory of reconsolidation, and (3) to which extent reconsolidation theory allows for precise and testable predictions. Parts of the figure were inspired by (27) and (28).

\section{Reconsolidation from an empirical perspective}

\section{Behavioral effects and reconsolidation as the underlying process}

Reconsolidation is most often inferred from behavioral results that illustrate susceptibility to an amnestic manipulation after a reminder (Fig. 2, (1)). Several criteria have been proposed to evaluate whether an observed behavioral effect may effectively represent reconsolidation interference (Table 1) (29-32). The question at hand is to which extent those criteria are met in studies that claim to demonstrate memory reconsolidation. Hardwicke (30) and Elsey et al. (29) provided valuable insights into this issue by evaluating published studies on reminder-dependent amnesia in human subjects across different types of memories and interventions (Table 1). To our knowledge, such an investigation has not yet been performed systematically for studies with non-human animals. Both reviews revealed that only a minority of reconsolidation studies had fully assessed the proposed criteria to infer reconsolidation interference from behavioral findings (see Elsey et al., Fig. 1 (29); and Hardwicke, Fig. 2.2 (30)). In addition, they identified several cases where some of the relevant criteria were explored, but not fulfilled (i.e., the observed results were not in line with what would be assumed 
on the basis of reconsolidation theory). Thus, many of the published human studies' positioning within a reconsolidation framework cannot be justified based on the adopted experimental designs.

\begin{tabular}{|c|c|c|c|c|}
\hline \multicolumn{3}{|c|}{ Criterion } & \multirow{2}{*}{$\begin{array}{c}\text { Hardwicke } \\
\text { (2016) }\end{array}$} & \multirow{2}{*}{$\begin{array}{l}\text { Elsey } \\
\text { (2018) }\end{array}$} \\
\hline & Required at procedural level & Predicted observation & & \\
\hline$A$ & $\begin{array}{l}\text { Do intervention at least one day } \\
\text { after initial encoding }\end{array}$ & NA & yes & no \\
\hline B & $\begin{array}{l}\text { Test Reminder x Manipulation } \\
\text { interaction }\end{array}$ & $\begin{array}{l}\text { Combination of reminder and } \\
\text { manipulation is crucial to } \\
\text { observe the effect }\end{array}$ & yes & yes \\
\hline C & Do manipulation after reminder & NA & yes & no \\
\hline D & $\begin{array}{l}\text { Vary reminder-manipulation } \\
\text { interval }\end{array}$ & $\begin{array}{l}\text { No effect when manipulation } \\
\text { takes place after termination of } \\
\text { putative reconsolidation period }\end{array}$ & yes & yes \\
\hline $\mathrm{E}$ & Perform STM and LTM test & $\begin{array}{l}\text { Effect of interest is observed at } \\
\text { LTM test, but not at STM test* }\end{array}$ & yes & yes \\
\hline $\mathrm{F}$ & Assess specificity of the effect & $\begin{array}{l}\text { Manipulation only affects the } \\
\text { allegedly reactivated memory }\end{array}$ & no & yes \\
\hline G & $\begin{array}{l}\text { Assess longevity of the effect } \\
\text { (e.g., recovery after time, } \\
\text { reinstatement, renewal, ...) }\end{array}$ & No recovery observed & yes & $\begin{array}{l}\text { not } \\
\text { required }\end{array}$ \\
\hline
\end{tabular}

Table 1. Fulfillment of various criteria has been suggested to be necessary for inferring reconsolidation interference from observed behavior. The last two columns indicate for each criterion whether it was included in the assessments by Hardwicke (2016) and Elsey et al. (2018).

(A) initial memory trace should have been consolidated (3), but see (33);

(B) provided by the same or a closely related study;

(C) pre-reactivation interventions leave more room for alternative explanations and are to be avoided if a study aims to demonstrate reconsolidation interference;

(D) the putative duration of this sensitivity time window varies between studies, ranging from one to six hours $(3,34)$;

(E) testing for behavioral effects should occur after the assumed reconsolidation process is completed and rule out the presence of any acute (e.g., drug) effect on behavior (long-term memory (LTM) test); additional testing shortly after the manipulation can serve as a negative control, given that interference should only become apparent after termination of the alleged reconsolidation period 
(short-term memory (STM) test; *except for post-reminder extinction learning, when similar STM and LTM performance is expected);

(F) the obtained effect should be specific to the reactivated memory

(G) behavioral effects should be permanent for a reconsolidation account to be valid $(12,30,32,35$, 36), although some claim that it is possible for reconsolidation interference to alter only a portion of the destabilized memory trace, allowing for at least partial recovery of memory performance $(29,37-$ 39).

In general - and this is by no means unique to the reconsolidation field - we should be cautious when concluding the engagement of an unobservable process based on an observed effect (e.g., a change in conditioned responding, task performance, or brain activity). A hypothetical example of such reverse inference (i.e., from observed effect to unobservable process) in the reconsolidation field can be as follows (adapted from (27)):

(1) In study A, the presentation of a retrieval cue followed by drug $X$ administration resulted in a decrease in fear responding compared to vehicle controls.

(2) In previous studies, an observed decrease in fear responding observed after reminder + drug $Y$ administration could be reasonably attributed to reconsolidation disruption.

(3) Therefore, the decrease in fear observed in study A also demonstrates reconsolidation disruption by drug $X$.

Although it has been acknowledged that reverse inference can be useful for scientific progress by suggesting novel hypotheses that can then be tested empirically, such inferences are only acceptable if the observation can occur if and only if the process of interest is engaged $(27,40)$. This implies that reminder-dependent behavioral effects cannot be equated to the presence of reconsolidation interference per se $(7,8,11)$. In addition, it should be acknowledged that behavioral studies can never prove the occurrence of a reconsolidation process, so caution is always required when making statements related to reconsolidation.

In sum, although adopting the terminology of 'reconsolidation' may be practically convenient, it has considerable disadvantages. First, reconsolidation has been claimed as an underlying mechanism for a range of behavioral observations in studies that were not actually designed to evaluate it as a candidate mechanism $(29,30)$. Second, inferring a neurobiological process based on observed behavioral results in general requires caution because such reverse inference is often unwarranted $(27,40)$. In the end, as described in the following section, such practices may pose serious problems for theoretical progress. 


\section{Behavioral effects and their relation to reconsolidation theory}

Theoretical progress is crucial to obtain a better understanding of when and how amnestic effects occur, which can greatly advance its proposed application in clinical settings. We maintain that the tendency to uncritically attribute reminder-dependent effects to reconsolidation interference has impeded such progress.

The vast majority of observed changes in memory performance after combining a reminder session with a manipulation has been situated within a reconsolidation context. Therefore, the effects observed in those studies have been assumed to share a destabilization/reconsolidation-dependent underlying process (Fig. 2, (1)). However, given the complex nature of amnesia and the unconditional and sometimes unjustified adherence to reconsolidation as the mechanism at play (as discussed in the previous section), 'reconsolidation' may well have been used to describe observations that in reality relied on a variety of processes (40). This implies that some of the assumptions embedded in reconsolidation theory may have been inferred from observations that were in fact the result of a process different from reconsolidation (Fig. 2, (2)). In addition, studies that were designed based on reconsolidation theory (e.g., by using certain reminder conditions that are assumed to induce memory destabilization and sensitivity to manipulation) may have failed to obtain an anticipated effect because the adopted procedure actually induced a process other than reconsolidation, to which different boundary conditions apply (Fig. 2, (2)).

Another way in which adherence to the notion of reconsolidation can be detrimental for theoretical progress is by slowing down the exploration of other viable explanations (40). For example, researchers may have been discouraged to investigate alternative explanations, such as statedependent learning, counterconditioning, or enhanced extinction, in the context of reminderdependent amnesia because this notion did not fit within the dominant reconsolidation framework. In addition, assuming reconsolidation as the underlying mechanism encourages researchers to neglect or explain away evidence against the occurrence of reconsolidation-based mechanisms (40). Indeed, as will be discussed in the next section, the flexible nature of proposed boundary conditions of the theory has enticed researchers to put forward such conditions to explain contradictory outcomes with recourse to reconsolidation theory.

\section{Reconsolidation from a theoretical perspective}

Whereas the previous section dealt with the inference of a reconsolidation process from empirical results and the potential pitfalls in linking behavioral observations to a neurobiological construct, the current section focuses on reconsolidation theory in itself and how it can and has been used to accommodate a wide range of empirical results. 


\section{Specificity as a key criterion of good theory}

A scientific theory is composed out of ideas that aim to predict and explain observed phenomena, and empirical research is required to test whether a theory actually fulfills this aim. During the iterative process of theory testing and refinement, a range of background or auxiliary assumptions are typically proposed to form the bridge between empirical observation and theory (26, 41). Those auxiliary assumptions are not part of a theory's core assumptions, but rather allow researchers to interpret experimental data in light of the theory; such as, for example, assumptions on how to interpret freezing responses in rodents or by considering the characteristics of a memory when assessing its malleability. Auxiliary assumptions may vary between experiments without jeopardizing the theory's core assumptions and can therefore be used to protect the theory's 'hard core' from refutation. However, it has been proposed that changes to those auxiliary assumptions are only adaptive if they can be confirmed empirically and if they allow to better explain or predict the phenomenon at hand (26).

An important aspect of a good theory is its specificity, allowing for the derivation of precise predictions that can be tested experimentally. However, it has been argued that most theories in the behavioral sciences are weak, given that they are too vague to allow for clear predictions (e.g., concerning the conditions under which the effect occurs or the magnitude of the effect) $(28,42-46)$. If a theory is poorly specified, it leaves room for researcher degrees of freedom and subjective interpretation of obtained results (i.e., flexibility), which may eventually result in the problem that basically any observation can be explained by the theory. The less specified the theory, the more researchers can rely on the use of (new) auxiliary assumptions. A failure to observe an effect can then be attributed to auxiliary assumptions, such as the proposal of a novel moderator, without questioning the theory's core assumptions (47). As a result, any empirical test addressing the theory has limited diagnostic value because it becomes unclear when a statistically significant effect falsifies or corroborates the theory.

The use of poorly specified theories further implies that the prior probability of any derived hypothesis being true is low (in other words, the predicted effect has a low base rate of occurrence), which has been shown to increase the chance of obtaining false positive results when using conventional criteria for null hypothesis testing (28). In addition, without the presence of a wellspecified theoretical framework, one cannot predict under which exact conditions an effect will (not) replicate and any replication failure does not allow us to draw conclusions about the accompanying theory. As such, it has been argued that the use of unspecific and flexible theories and/or auxiliary assumptions plays a major role in replicability issues in general $(28,46,48,49)$. 


\section{A principled evaluation of reconsolidation theory}

Apart from submitting a theory to empirical testing, it can thus also be criticized conceptually by assessing whether it adheres to certain criteria. In the current section, we will evaluate reconsolidation theory by discussing its specificity (Fig. 2, (3)), a key aspect of good theory, as explained above.

Reconsolidation theory posits that combining a reminder session with a manipulation can induce a change in mnemonic performance. The theory's core assumptions entail that (1) the reminder session can result in memory destabilization (making the memory temporally sensitive to modification) and the requirement of reconsolidation, and (2) the manipulation interferes with the reconsolidation process to induce the observable behavioral effect; or some cases, it is assumed that the presented information becomes incorporated in the destabilized memory trace, resulting in an updated memory $(36,50)$. In the current literature, empirical testing of the predictions derived from reconsolidation theory employs a wide range of procedures with different reminder sessions and manipulations, and the theory predicts that an effect can be observed in some cases, without clearly specifying the conditions under which the effect should occur or not (28). For instance, it appears that there is considerable flexibility in the type of manipulations that are deemed effective. Reconsolidation, like consolidation, is assumed to entail protein synthesis-dependent memory storage. In line with the involvement of signalling pathways that are thought to coordinate protein synthesis, several pharmacological and genetic manipulations related to such pathways have been used to interfere with alleged memory reconsolidation (e.g. targeting noradrenergic or glutamatergic receptors, kinases, transcription factors or immediate-early gene expression). However, a wide range of manipulations that have not been clearly linked to those signalling pathways have nevertheless been claimed to also interfere with reconsolidation (e.g., targeting serotonergic, cannabinoid, histamine, oxytocin, adenosine, GABA and opioid receptors, or various types of hormones, peptides, cytokines, and toxins) (51). This illustrates that the core assumptions of reconsolidation theory leave quite some room for labeling diverse reminder-dependent behavioral effects as being instances of reconsolidation interference, rather than providing a clearly demarcated scope. In other words, any study combining a reminder and a manipulation and showing a change in behavior is typically seen as in line with reconsolidation theory, whereas a study that fails to observe such an effect does not necessarily question the theory's core assumptions; it merely reflects that the adopted procedure (or procedural details) cannot be used to obtain the effect (28). Hence, auxiliary assumptions, mostly consisting of proposed moderators or boundary conditions on memory destabilization, can be used to provide a seemingly acceptable explanation for many results. As illustrated below, this state of affairs is problematic due to the flexible properties of the proposed boundary conditions themselves. 


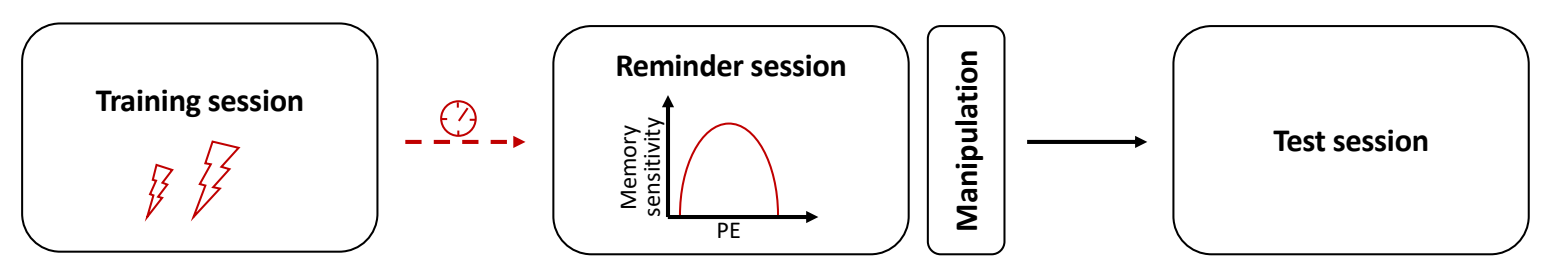

Figure 3. Commonly-proposed boundary conditions on memory destabilization and subsequent reconsolidation include the strength of training, the time interval between training and the reminder session, and the amount of prediction error (PE) induced during the reminder session.

Studies that parametrically manipulated proposed boundary conditions on reconsolidation have provided important insights into the conditions required for obtaining reminder-dependent behavioral effects. As such, the intensity and type of training, the duration of the trainingmanipulation interval, the amount of novel information during the reminder session, and the occurrence of extinction learning have received empirical support (see Fig. 3) (52-61). However, it should be acknowledged that most of those moderators rest on mixed empirical evidence, because not all studies have found support for their existence, and the exact characteristics of the boundary conditions seem to differ between studies. For example, the time interval between training and reminder session (i.e., memory age) over which memories remain sensitive to a post-reminder manipulation varies substantially between studies (e.g., sensitivity up to 2 days after training, (62); up to 3 but not 7 days, (63); up to 7 but not 14 days, (56); up to 21 but not 56 days, (59), and no evidence for such temporal gradient was found in other studies when the reminder took place 1 or 14 days after training (3) or 3, 15 or 45 days after training (64) (i.e., memory sensitivity did not change over time). Another widely accepted boundary condition is the strength of training, which is typically manipulated by varying the intensity, duration, or number of presentations of the unconditioned stimulus (in case of classical conditioning) or the length of the training session (61), through pre-learning stress induction (65), or by pharmacologically enhancing noradrenergic activity around the time of learning (66). Yet, the classification of a training session as 'weak' or 'strong' varies considerably between studies. For example, a foot shock of $.8 \mathrm{~mA}$ has been used in mice to induce strong - difficult to destabilize - contextual fear memory (54), whereas roughly the same intensity (two shocks of $.75 \mathrm{~mA}$ ) has been used as the 'weak training' - easy to destabilize - condition in another study (59). The mixed observed results regarding the role of boundary conditions have led to the notion that memoryrelated factors (such as age and strength) may operate as boundary conditions only under certain circumstances (e.g., depending on several other factors of the adopted experimental procedure). In addition, it has been illustrated that boundary conditions interact with each other and that they can 
be overcome, e.g., by modifying the duration of the reminder session, prolonging the learningreminder interval, or adding novel information to the reminder session (59-61). Thus, although the role of memory age and strength in memory sensitivity has been illustrated experimentally, it is unclear when exactly those factors will or will not prevent alleged memory destabilization.

Apart from memory age and strength, behavioral studies have suggested that the occurrence of memory malleability relies on the presence of novel information or surprise during the reminder session and depends on the degree of mismatch between predicted and observed events (i.e., prediction error). It has been put forward that there is only a small window during which a memory is assumed to destabilize; i.e. under conditions that evoke an optimal degree of prediction error (67) (Fig. 3). Nevertheless, there is no specification of what constitutes an optimal degree of mismatch for achieving the assumed destabilization, partly because this is presumed to be highly dependent on a range of other factors such as the preceding training conditions. In fact, finding the appropriate reminder conditions to achieve memory sensitivity seems to be a matter of trial and error. For example, when considering published rodent studies using comparable contextual fear conditioning procedures, a wide variety of different durations of unreinforced context re-exposure has been found to allow for a behavioral change by post-reminder treatment (51), illustrating that optimal conditions can vary considerably between studies.

An additional difficulty is that prediction error cannot be measured directly, nor is it clear how it should be implemented during the reminder session. This uncertainty has contributed to the flexibility in the adopted experimental designs that have been claimed to induce prediction error. In case of classical conditioning, a mismatch between initial learning and reminder conditions is typically effectuated by omitting the predicted outcome during presentation of the conditioned stimulus (e.g., presenting the conditioned cue without the shock with which it was paired during training). The degree of prediction error has been manipulated by varying the amount of unreinforced trials or the duration of the unreinforced reminder session (for more examples of prediction error induction see $(68,69))$. For other types of memories, such as episodic memory, it is less straightforward how to implement prediction error in the reminder session. Terminating a previously-watched video fragment before it has finished, ending a test trial before the participant has had the chance to perform the response, recalling objects that were stored in a basket by re-exposure to the same basket have all been assumed to induce prediction error because the reminder session is a partial, but not full, recap of the training session (70). On the other hand, there are several published studies that were not actually designed to induce prediction error given that training and reminder conditions were very similar, such as a reinforced retrieval trial identical to the training session (71), presentation 
of images from a previously watched trauma film (72), or a simple recall task (73), and yet, a reconsolidation-dependent effect was reported.

Overall, the commonly proposed boundary conditions on memory destabilization cannot be clearly operationalized (i.e., they are abstract concepts that do not lend themselves to being quantified by empirical observation) or implemented (i.e., the application of each boundary condition varies considerably between studies). Due to those characteristics, boundary conditions cannot be translated into specific criteria that can be used to make precise predictions about when memories will become susceptible to change. Rather, they contribute to the flexibility that allows the linking of many behavioral observations to reconsolidation theory. Indeed, in practice, failures to observe an anticipated reconsolidation-dependent effect have typically been attributed to the unintended presence of (unknown) boundary conditions on memory destabilization (e.g., the memory might have been too strong or the amount of prediction error might have been inappropriate, also in our own work $(24,74))$. The proposed moderators of the effect are thus used to protect reconsolidation theory from being contradicted and can allow researchers to explain away inherently conflicting findings with recourse to reconsolidation theory. This, in turn, has made it difficult to conclude whether an empirical observation is consistent or inconsistent with the theory.

\section{Future directions}

\section{Distinguishing observed behavior from unobservable underlying processes}

As previously suggested for this domain $(14,35)$ and for empirical behavioral research in general $(40,75)$, we maintain that unobservable mechanisms, such as reconsolidation interference, should be clearly distinguished from observed behavioral effects. As such, the term reconsolidation should exclusively be used to refer to a theoretical concept, whereas behavioral effects should be expressed purely in terms of preceding environmental changes and the accompanying observations $(35,40)$; for example, "a decrease in fear responding resulting from unreinforced presentation of a conditioned cue followed by anisomycin injection". In this regard, the term 'reminder-dependent amnesia' may be a more appropriate label to refer to these behavioral effects given that it remains uncommitted to any underlying process. If reconsolidation interference is raised as a possible neurobiological process underlying an observed behavior, the suggested criteria outlined above (see Table 1) need to be considered and alternative explanations ought to be ruled out or acknowledged. We do not intend to argue that all research on reminder-dependent manipulations should necessarily aim to test whether or not reconsolidation can account for the results. We do claim that if a study is presented within a reconsolidation framework, it should be appropriately designed (in accordance 
with the proposed list of criteria) to investigate reconsolidation interference as a possible mechanism, and, in any case, behavioral effects should never be equalized with the occurrence of a reconsolidation process.

\section{Increasing the precision (and falsifiability) of theory and hypotheses}

Enhancing awareness and acknowledging the flexibility inherent to reconsolidation theory can be helpful and discourage researchers from (mis)using boundary conditions to explain most results with recourse to reconsolidation theory. Furthermore, it can encourage the adoption of a more critical perspective on reconsolidation theory, and enhance openness to alternative explanations. However, merely acknowledging the limitations of reconsolidation theory will not in itself lead to greater progress. Rather, the ultimate aim should be to formulate theories in a more specific way. This in turn allows for the derivation of specific hypotheses that are able to challenge and improve the theory in case of disconfirmation by empirical data. An emerging approach to make more precise predictions is the use of computational models $(28,44)$, but see $(76)$. Rare examples of such approaches exist in the reconsolidation literature (77-79), with each of the proposed computational models focusing on a specific subset of data within the reconsolidation field. However, as illustrated in the next paragraph, building such a concrete theoretical framework for reminder-dependent effects may be challenging at present, given that some of the basic findings (e.g., those that have inspired reconsolidation theory) have proven difficult to replicate.

\section{Establishing a reliable empirical base to inform theory development}

Some researchers found amnesia when electroconvulsive shock was given after re-exposure to a previously conditioned tone, e.g., $(2,80)$, whereas others did not, e.g., $(19,20)$. Similarly, influential findings from studies with human participants showing reminder-dependent attenuation of fear memories, e.g., (81-83), or episodic memories, e.g., (84), have failed to replicate, e.g., (16, 21, 25). Studies on post-retrieval extinction have also yielded mixed results, with many of them showing fear memory attenuation in humans and rodents, e.g., $(36,85-88)$, but others failing to obtain such effects, e.g., $(18,23,83)$. Importantly, in many cases the attempted replication experiments adhered to the methodology of previously-published studies as closely as possible and yet they failed to observe the expected outcome $(18,22,23,30)$. Such failures to exactly replicate can either be attributed to the occurrence of false positive results in the original studies, the replication results being false negatives, or the absence of a clear understanding of the phenomenon. Replication failures can inspire a formal investigation of possible moderators to increase insight in the factors that may have led to those negative results and generally improve our understanding of when an effect can be 
observed, such as, for example, investigating the role of interindividual (89) or subtle environmental (90) differences.

Given the presence of several contradictory findings within the field, it is valuable to maximize research credibility. For instance, systematically and critically reviewing published results and performing well-powered multi-site replication studies of previously published basic findings could help to gain more insight into whether reported results reflect true effects (i.e., assess reliability) and whether the effects hold over time, in different lab environments, populations, etc. (i.e., assess generalizability). In addition, it might be useful to examine the extent to which original conclusions hold when using different analysis strategies (i.e., assess robustness). As previously indicated, doing research in the absence of a well-specified theoretical framework entails a relatively high risk of obtaining false positive results. If it turns out that (some of) the obtained effects are not reliable, robust, and/or have limited generalizability, it does not seem particularly useful to build a theoretical framework to explain those findings. As briefly illustrated below, several methodologically-oriented tools can promote the credibility of the conclusions that are drawn from empirical research.

Making data and analysis scripts publicly available or preregistering hypotheses, methods and planned analyses (i.e., archiving them in a public repository prior to data collection and under an embargo specified by the author) (91-93) increases research transparency. Preregistration enhances researchers' ability to judge the credibility of published results and protects researchers from confirmation and hindsight bias. Integrating preregistration in the publication process in the format of a Registered Report moreover ensures that studies are published based on the soundness of the proposed research questions and quality of the methods regardless of the obtained outcome (94). Research has shown that the use of preregistration and Registered Reports goes hand in hand with a decline in the percentage of published studies reporting statistically significant ('positive') results, possibly by decreasing the use of suboptimal research practices $(95,96)$. Of note, preregistration does not guarantee that analysis plans and hypotheses are based on rational and justifiable decisions, and in itself cannot make up for bad theory.

Research transparency can be further increased by adopting additional method-oriented, as well as more theory-oriented approaches; first, by reporting the sensitivity of obtained results to arbitrary decisions made during data processing. Researchers typically choose from a range of several reasonable data processing options (e.g., which outliers to exclude, how to categorize or combine variables, etc.), and it has been proposed that research transparency and the insight in robustness of statistical results can be improved by doing a 'multiverse' analysis (97) or 'specification curve' analysis (98), in which the results for all reasonable choices during data processing are reported. Second, transparency on a theory level could be enhanced if researchers indicate under which scenario(s) the 
proposed hypothesis would be disconfirmed, and whether that would challenge the underlying theory or whether changes to the theory should be made (42). Such specification would allow for a clearer judgement of the extent to which theory can be falsified by empirical testing $(91,99)$ and it may avoid that predictions will almost certainly be confirmed by empirical observation.

\section{Conclusion}

Successful clinical application of reminder-dependent memory interference currently seems more hype than reality given the abundance of mixed results and elusive null findings. In order to promote our knowledge of reminder-dependent effects, we have highlighted some of the current theoretical issues and suggested possible solutions. Caution is required when inferring reconsolidation from behavioral results and when using reconsolidation theory to predict and account for such data. In addition, awareness should be raised regarding the lack of specificity in reconsolidation theory and the flexibility of boundary conditions. We propose that every single step towards increasing transparency at a methodological and theoretical level would be beneficial for scientific progress, and, ultimately, for more fruitful translational efforts. 


\section{References}

1. T. Beckers, M. Kindt, Memory reconsolidation interference as an emerging treatment for emotional disorders: Strengths, limitations, challenges, and opportunities, Annu Rev Clin Psychol 13, 99-121 (2017).

2. J. R. Misanin, R. R. Miller, D. J. Lewis, Retrograde amnesia produced by electroconvulsive shock after reactivation of a consolidated memory trace, Science 160, 554-555 (1968).

3. K. Nader, G. E. Schafe, J. Le Doux, Fear memories require protein synthesis in the amygdala for reconsolidation after retrieval, Nature 406, 722-6 (2000).

4. J. Przybyslawski, S. J. Sara, Reconsolidation of memory after its reactivation, Behav. Brain Res. 84, 241-246 (1997).

5. M. T. J. Exton-McGuinness, J. L. C. Lee, A. C. Reichelt, Updating memories--the role of prediction errors in memory reconsolidation, Behav. Brain Res. 278, 375-384 (2015).

6. K. Nader, Reconsolidation and the dynamic nature of memory, Cold Spring Harb Perspect Biol 7, a021782 (2015).

7. J. M. Alfei, R. I. Ferrer Monti, V. A. Molina, D. De Bundel, L. Luyten, T. Beckers, Generalization and recovery of post-retrieval amnesia, Journal of Experimental Psychology: General (2020).

8. J. M. Alfei, H. De Gruy, D. De Bundel, L. Luyten, T. Beckers, Apparent reconsolidation interference without generalized amnesia, Progress in Neuropsychopharmacology \& Biological Psychiatry 108 (2021), doi:10.1016/j.pnpbp.2020.110161.

9. Y. Boddez, A. Moors, G. Mertens, J. De Houwer, Tackling fear: Beyond associative memory activation as the only determinant of fear responding, Neuroscience \& Biobehavioral Reviews 112, 410-419 (2020).

10. E. N. Cahill, M. A. Wood, B. J. Everitt, A. L. Milton, The role of prediction error and memory destabilization in extinction of cued-fear within the reconsolidation window, Neuropsychopharmacol. 44, 1762-1768 (2019).

11. P. Gisquet-Verrier, J. F. Lynch, P. Cutolo, D. Toledano, A. Ulmen, A. M. Jasnow, D. C. Riccio, Integration of new information with active memory accounts for retrograde amnesia: A challenge to the consolidation/reconsolidation hypothesis?, The Journal of Neuroscience 35, 11623-11633 (2015).

12. K. M. Lattal, T. Abel, Behavioral impairments caused by injections of the protein synthesis inhibitor anisomycin after contextual retrieval reverse with time, Proceedings of the National Academy of Sciences 101, 4667-4672 (2004).

13. D. C. Riccio, P. M. Millin, A. R. Bogart, Reconsolidation: A brief history, a retrieval view, and some recent issues, Learning \& Memory 13, 536-544 (2006).

14. J. W. Rudy, Anisomycin and the reconsolidation hypothesis, Learning \& Memory 13, 1-3 (2006).

15. J. C. Biedenkapp, J. W. Rudy, Context memories and reactivation: Constraints on the reconsolidation hypothesis., Behavioral Neuroscience 118, 956-964 (2004). 
16. M. G. N. Bos, T. Beckers, M. Kindt, Noradrenergic blockade of memory reconsolidation: A failure to reduce conditioned fear responding, Frontiers in Behavioral Neuroscience 8 (2014), doi:10.3389/fnbeh.2014.00412.

17. M. B. L. Careaga, P. A. Tiba, S. M. Ota, D. Suchecki, Pre-test metyrapone impairs memory recall in fear conditioning tasks: Lack of interaction with $\beta$-adrenergic activity, Front. Behav. Neurosci. 9 (2015), doi:10.3389/fnbeh.2015.00051.

18. A. Chalkia, N. Schroyens, L. Leng, N. Vanhasbroeck, A.-K. Zenses, L. Van Oudenhove, T. Beckers, No persistent attenuation of fear memories in humans: A registered replication of the reactivationextinction effect, Manuscript submitted for publication (2020).

19. R. G. Dawson, J. L. McGaugh, Electroconvulsive shock effects on a reactivated memory trace: Further examination, Science 166, 525-527 (1969).

20. H. Elahi, V. Hong, J. E. Ploski, Electroconvulsive shock does not impair the reconsolidation of cued and contextual pavlovian threat memory, IJMS 21, 7072 (2020).

21. T. E. Hardwicke, M. Taqi, D. R. Shanks, Postretrieval new learning does not reliably induce human memory updating via reconsolidation, Proceedings of the National Academy of Sciences 113, 52065211 (2016).

22. L. Luyten, A. E. Schnell, N. Schroyens, T. Beckers, Lack of drug-induced post-retrieval amnesia for auditory fear memories in rats, BMC Biol 19, 17 (2021).

23. L. Luyten, T. Beckers, A preregistered, direct replication attempt of the retrieval-extinction effect in cued fear conditioning in rats, Neurobiology of Learning and Memory 144, 208-215 (2017).

24. N. Schroyens, J. M. Alfei, A. E. Schnell, L. Luyten, T. Beckers, Limited replicability of drug-induced amnesia after contextual fear memory retrieval in rats, Neurobiology of Learning and Memory 166, 107105 (2019).

25. J. Thome, G. Koppe, S. Hauschild, L. Liebke, C. Schmahl, S. Lis, M. Bohus, M. Baudry, Ed. Modification of fear memory by pharmacological and behavioural interventions during reconsolidation, PLOS ONE 11, e0161044 (2016).

26. I. Lakatos, in Can theories be refuted?, (Springer, Dordrecht, 1976).

27. R. Poldrack, Can cognitive processes be inferred from neuroimaging data?, Trends in Cognitive Sciences 10, 59-63 (2006).

28. K. Oberauer, S. Lewandowsky, Addressing the theory crisis in psychology, Psychon Bull Rev 26, 1596-1618 (2019).

29. J. W. B. Elsey, V. A. Van Ast, M. Kindt, Human memory reconsolidation: A guiding framework and critical review of the evidence., Psychological Bulletin 144, 797-848 (2018).

30. T. E. Hardwicke, thesis, University College London (2016).

31. M. C. W. Kroes, D. Schiller, J. Le Doux, E. A. Phelps, Translational approaches targeting reconsolidation, Current topics in behavioral neurosciences 28, 197-230 (2016). 
32. N. C. Tronson, J. R. Taylor, Molecular mechanisms of memory reconsolidation, Nat Rev Neurosci 8, 262-275 (2007).

33. Y. Dudai, M. Eisenberg, Rites of passage of the engram: Reconsolidation and the lingering consolidation hypothesis, Neuron 44, 93-100 (2004).

34. S. G. Bustos, H. Maldonado, V. A. Molina, Midazolam disrupts fear memory reconsolidation, Neuroscience 139, 831-842 (2006).

35. R. R. Miller, Failures of memory and the fate of forgotten memories, Neurobiology of Learning and Memory , 107426 (2021).

36. M.-H. Monfils, K. K. Cowansage, E. Klann, J. E. LeDoux, Extinction-reconsolidation boundaries: Key to persistent attenuation of fear memories, Science 324, 951-955 (2009).

37. O. B. Amaral, R. Osan, R. Roesler, A. B. L. Tort, A synaptic reinforcement-based model for transient amnesia following disruptions of memory consolidation and reconsolidation, Hippocampus 18, 584-601 (2008).

38. P. E. Gold, R. A. King, Retrograde amnesia: storage failure versus retrieval failure, Psychological Review 81, 465-469 (1974).

39. K. Nader, S.-H. Wang, Fading in, Learning \& Memory 13, 530-535 (2006).

40. J. De Houwer, Why the cognitive approach in psychology would profit from a functional approach and vice versa, Perspectives on Psychological Science 6, 202-209 (2011).

41. K. R. Popper, The logic of scientific discovery, Systematic Zoology 26 (1977), doi:https://doi.org/10.2307/2412687.

42. E. I. Fried, Lack of theory building and testing impedes progress in the factor and network literature, Psychological Inquiry (in press).

43. K. Gieseler, D. D. Loschelder, M. Friese, in Social psychology in action, (Springer Nature, Switzerland, 2019).

44. I. Grahek, M. Schaller, J. L. Tackett, Anatomy of a psychological theory: Integrating constructvalidation and computational-modeling methods to advance theorizing, Perspect Psychol Sci , 174569162096679 (2021).

45. P. E. Meehl, Appraising and amending theories: The strategy of Lakatosian defense and two principles that warrant It, Psychological Inquiry 1, 108-141 (1990).

46. A. Szollosi, C. Donkin, Arrested theory development: The misguided distinction between exploratory and confirmatory research, Perspectives on Psychological Science (in press).

47. S. J. Gershman, How to never be wrong, Psychon Bull Rev 26, 13-28 (2019).

48. S. B. Klein, What can recent replication failures tell us about the theoretical commitments of psychology?, Theory \& Psychology 24, 326-338 (2014).

49. D. Trafimow, B. D. Earp, Badly specified theories are not responsible for the replication crisis in social psychology: Comment on Klein, Theory \& Psychology 26, 540-548 (2016). 
50. J. Haubrich, A. P. Crestani, L. F. Cassini, F. Santana, R. O. Sierra, L. de O. Alvares, J. A. Quillfeldt, Reconsolidation allows fear memory to be updated to a less aversive level through the incorporation of appetitive information, Neuropsychopharmacology 40, 315-326 (2015).

51. N. Schroyens, E. L. Sigwald, W. Van Den Noortgate, T. Beckers, L. Luyten, Reactivation-dependent amnesia for contextual fear memories: Evidence for publication bias, eNeuro 8, ENEURO.010820.2020 (2021).

52. M. Eisenberg, Stability of retrieved memory: Inverse correlation with trace dominance, Science 301, 1102-1104 (2003).

53. A. Hupbach, R. Gomez, L. Nadel, Episodic memory updating: The role of context familiarity, Psychon Bull Rev 18, 787-797 (2011).

54. C. Kwak, J.-H. Choi, J. T. Bakes, K. Lee, B.-K. Kaang, Effect of intensity of unconditional stimulus on reconsolidation of contextual fear memory, Korean J Physiol Pharmacol 16, 293 (2012).

55. E. Merlo, A. L. Milton, Z. Y. Goozee, D. E. Theobald, B. J. Everitt, Reconsolidation and extinction are dissociable and mutually exclusive processes: Behavioral and molecular evidence, Journal of Neuroscience 34, 2422-2431 (2014).

56. M. H. Milekic, C. M. Alberini, Temporally graded requirement for protein synthesis following memory reactivation, Neuron 36, 521-525 (2002).

57. M. E. Pedreira, L. M. Pérez-Cuesta, H. Maldonado, Mismatch between what is expected and what actually occurs triggers memory reconsolidation or extinction, Learn. Mem. 11, 579-585 (2004).

58. D. Sevenster, T. Beckers, M. Kindt, Prediction error demarcates the transition from retrieval, to reconsolidation, to new learning, Learning \& Memory 21, 580-584 (2014).

59. A. Suzuki, Memory reconsolidation and extinction have distinct temporal and biochemical signatures, Journal of Neuroscience 24, 4787-4795 (2004).

60. S.-H. Wang, L. de Oliveira Alvares, K. Nader, Cellular and systems mechanisms of memory strength as a constraint on auditory fear reconsolidation, Nature Neuroscience 12, 905-912 (2009).

61. B. D. Winters, M. C. Tucci, M. DaCosta-Furtado, Older and stronger object memories are selectively destabilized by reactivation in the presence of new information, Learning \& Memory 16, 545-553 (2009).

62. O. O. Litvin, K. V. Anokhin, Mechanisms of memory reorganization during retrieval of acquired behavioral experience in chicks: The effects of protein synthesis inhibition in the brain, Neuroscience and Behavioral Physiology , 8 (2000).

63. M. Eisenberg, Y. Dudai, Reconsolidation of fresh, remote, and extinguished fear memory in medaka: Old fears don't die, European Journal of Neuroscience 20, 3397-3403 (2004).

64. J. Debiec, J. E. LeDoux, K. Nader, Cellular and systems reconsolidation in the hippocampus, Neuron 36, 527-538 (2002).

65. P. J. Espejo, V. Ortiz, I. D. Martijena, V. A. Molina, Stress-induced resistance to the fear memory labilization/reconsolidation process. Involvement of the basolateral amygdala complex, Neuropharmacology 109, 349-356 (2016). 
66. L. Gazarini, C. A. J. Stern, R. R. Piornedo, R. N. Takahashi, L. J. Bertoglio, PTSD-like memory generated through enhanced noradrenergic activity is mitigated by a dual step pharmacological intervention targeting its reconsolidation, International Journal of Neuropsychopharmacology 18 (2015), doi:10.1093/ijnp/pyu026.

67. L. F. Cassini, C. R. Flavell, O. B. Amaral, J. L. C. Lee, On the transition from reconsolidation to extinction of contextual fear memories, Learning \& Memory 24, 392-399 (2017).

68. J. M. Alfei, R. I. Ferrer Monti, V. A. Molina, A. M. Bueno, G. P. Urcelay, Prediction error and trace dominance determine the fate of fear memories after post-training manipulations, Learning \& Memory 22, 385-400 (2015).

69. L. Díaz-Mataix, R. C. Ruiz Martinez, G. E. Schafe, J. E. LeDoux, V. Doyère, Detection of a temporal error triggers reconsolidation of amygdala-dependent memories, Curr. Biol. 23, 467-472 (2013).

70. A. H. Sinclair, M. D. Barense, Prediction error and memory reactivation: How incomplete reminders drive reconsolidation, Trends in Neurosciences 42, 727-739 (2019).

71. S. Duvarci, K. Nader, Characterization of fear memory reconsolidation, J. Neurosci. 24, 92699275 (2004).

72. E. L. James, M. B. Bonsall, L. Hoppitt, E. M. Tunbridge, J. R. Geddes, A. L. Milton, E. A. Holmes, Computer game play reduces intrusive memories of experimental trauma via reconsolidation-update mechanisms, Psychol Sci 26, 1201-1215 (2015).

73. J. C. K. Chan, J. A. LaPaglia, Impairing existing declarative memory in humans by disrupting reconsolidation, Proc Natl Acad Sci USA 110, 9309-9313 (2013).

74. N. Schroyens, T. Beckers, M. Kindt, In search for boundary conditions of reconsolidation: A failure of fear memory interference, Frontiers in Behavioral Neuroscience 11 (2017), doi:10.3389/fnbeh.2017.00065.

75. N. Vahey, R. Whelan, The functional-cognitive framework as a tool for accelerating progress in cognitive neuroscience: On the benefits of bridging rather than reducing levels of analyses, Int $J$ Psychol 51, 45-49 (2016).

76. K. Fiedler, What constitutes strong psychological science? The (neglected) role of diagnosticity and a priori theorizing, Perspect Psychol Sci 12, 46-61 (2017).

77. S. J. Gershman, M.-H. Monfils, K. A. Norman, Y. Niv, The computational nature of memory modification, eLife 6, e23763 (2017).

78. R. Osan, A. B. L. Tort, O. B. Amaral, G. Cymbalyuk, Ed. A mismatch-based model for memory reconsolidation and extinction in attractor networks, PLOS ONE 6, e23113 (2011).

79. P. B. Sederberg, S. J. Gershman, S. M. Polyn, K. A. Norman, Human memory reconsolidation can be explained using the temporal context model, Psychon Bull Rev 18, 455-468 (2011).

80. A. M. Schneider, W. Sherman, Amnesia: A Function of the temporal relation of footshock to electroconvulsive Shock, Science 159, 219-221 (1968).

81. M. Kindt, M. Soeter, B. Vervliet, Beyond extinction: Erasing human fear responses and preventing the return of fear, Nature Neuroscience 12, 256-258 (2009). 
82. D. Sevenster, T. Beckers, M. Kindt, Retrieval per se is not sufficient to trigger reconsolidation of human fear memory, Neurobiology of Learning and Memory 97, 338-345 (2012).

83. M. Soeter, M. Kindt, Disrupting reconsolidation: Pharmacological and behavioral manipulations, Learning \& Memory 18, 357-366 (2011).

84. M. P. Walker, T. Brakefield, J. Allan Hobson, R. Stickgold, Dissociable stages of human memory consolidation and reconsolidation, Nature 425, 616-620 (2003).

85. T. Agren, J. Engman, A. Frick, J. Bjorkstrand, E.-M. Larsson, T. Furmark, M. Fredrikson, Disruption of reconsolidation erases a fear memory trace in the human amygdala, Science 337, 1550-1552 (2012).

86. C. R. Flavell, D. J. Barber, J. L. C. Lee, Behavioural memory reconsolidation of food and fear memories, Nat Commun 2, 504 (2011).

87. J. Liu, L. Zhao, Y. Xue, J. Shi, L. Suo, Y. Luo, B. Chai, C. Yang, Q. Fang, Y. Zhang, Y. Bao, C. L. Pickens, L. Lu, An unconditioned stimulus retrieval extinction procedure to prevent the return of fear memory, Biological Psychiatry 76, 895-901 (2014).

88. P. Rao-Ruiz, D. C. Rotaru, R. J. van der Loo, H. D. Mansvelder, O. Stiedl, A. B. Smit, S. Spijker, Retrieval-specific endocytosis of GluA2-AMPARs underlies adaptive reconsolidation of contextual fear, Nat Neurosci 14, 1302-1308 (2011).

89. J. Shumake, C. Jones, A. Auchter, M.-H. Monfils, Data-driven criteria to assess fear remission and phenotypic variability of extinction in rats, Phil. Trans. R. Soc. B 373, 20170035 (2018).

90. N. Schroyens, C. L. Bender, J. M. Alfei, V. A. Molina, L. Luyten, T. Beckers, Post-weaning housing conditions influence freezing during contextual fear conditioning in adult rats, Behavioural Brain Research 359, 172-180 (2019).

91. D. Lakens, The value of preregistration for psychological science: A conceptual analysis, Japanese Psychological Review 62, 221-230 (2019).

92. B. A. Nosek, C. R. Ebersole, A. C. DeHaven, D. T. Mellor, The preregistration revolution, Proc Natl Acad Sci USA 115, 2600-2606 (2018).

93. E.-J. Wagenmakers, R. Wetzels, D. Borsboom, H. L. J. van der Maas, R. A. Kievit, An agenda for purely confirmatory research, Perspect Psychol Sci 7, 632-638 (2012).

94. C. D. Chambers, Registered Reports: A new publishing initiative at Cortex, Cortex 49, 609-610 (2013).

95. R. M. Kaplan, V. L. Irvin, S. Garattini, Ed. Likelihood of Null Effects of Large NHLBI Clinical Trials Has Increased over Time, PLOS ONE 10, e0132382 (2015).

96. A. M. Scheel, M. Schijen, D. Lakens, An excess of positive results: Comparing the standard Psychology literature with Registered Reports, Advances in Methods and Practices in Psychological Science (in press), doi:10.31234/osf.io/p6e9c.

97. S. Steegen, F. Tuerlinckx, A. Gelman, W. Vanpaemel, Increasing transparency through a multiverse analysis, Perspect Psychol Sci 11, 702-712 (2016). 
98. U. Simonsohn, J. P. Simmons, L. D. Nelson, Specification curve: Descriptive and inferential statistics on all reasonable specifications, SSRN Journal (2015), doi:10.2139/ssrn.2694998.

99. W. Vanpaemel, The Really Risky Registered Modeling Report: Incentivizing strong tests and HONEST modeling in cognitive science, Comput Brain Behav 2, 218-222 (2019). 